\title{
On a reflexivity-preserving family of cardinality-based fuzzy comparison measures
}

\author{
Klaas Bosteels*, Etienne E. Kerre \\ Fuzziness and Uncertainty Modelling Research Group \\ Department of Applied Mathematics and Computer Science \\ Ghent University, Krijgslaan 281 (S9), B-9000 Gent, Belgium
}

\begin{abstract}
In this paper, we generalize and extend previous work on a triparametric family of cardinality-based fuzzy similarity measures. More specifically, we (i) generalize this family to a broader family of fuzzy comparison measures, (ii) summarize previously obtained results regarding this family and fill up a few gaps by adding some novel results, (iii) compare our parametric family with an already existing one, and (iv) elaborate on the ranking invariance between certain members of the presented triparametric family of cardinality-based fuzzy comparison measures.
\end{abstract}

Key words: Fuzzy sets, Comparison, Compatibility, Inclusion, Resemblance, Similarity, Reflexivity, Ranking invariance

\section{Introduction}

Many applications in information sciences require a way to compare fuzzy sets. In particular, a measure to determine the similarity between two given fuzzy sets is a key component of many algorithms in this domain (e.g. $[1,13,16,19]$ ). Since different applications have different needs, it makes sense to study systematic ways of constructing and analyzing such measures. Motivated by this insight, we presented a systematic way of generating cardinality-based fuzzy similarity measures in [4]. More precisely, we introduced a general form that depends on two parameters, together with constraints on these parameters

* Corresponding author. Tel.: +32-9-264-9639; fax: +32-9-264-4995.

Email addresses: klaas.bosteels@ugent.be (Klaas Bosteels), etienne.kerre@ugent.be (Etienne E. Kerre). 
that ensure various properties of the generated measures. Later on, we generalized this biparametric family of cardinality-based fuzzy similarity measures to a triparametric one, and provided rewritten versions of the previously introduced constraints, as well as several additional constraints [6]. In this paper, we further generalize the triparametric family to a broader family of fuzzy comparison measures, and we summarize previously obtained results and constraints. Moreover, we fill up a few gaps by adding some novel constraints, and we compare the presented triparametric family with the parametric family of cardinality-based fuzzy comparison measures introduced in [12]. In particular, we provide both theoretical and empirical evidence showing that the reflexivity-preserving nature of our parametric family can be considered an advantage over the alternative one. In order to explain the somewhat surprising results of the experiment that led to the empirical evidence for this claim, we also take a closer look at the ranking invariance between certain members of the presented family of fuzzy comparison measures.

\section{Preliminaries}

\subsection{Basic notations}

In the remainder of this paper, $U$ always denotes a nonempty finite universe. We use the notation $\mathcal{F}(U)$ for the class of fuzzy sets in $U$, i.e., the (crisp) set of all $U \rightarrow[0,1]$ mappings. For two fuzzy sets $A$ and $B$ in $U$, we write $A \subseteq B$ iff $A(u) \leq B(u)$ for all $u \in U$, and $A=B$ iff $A \subseteq B \wedge B \subseteq A$. Furthermore, we let $\operatorname{supp} A$ denote the support of a fuzzy set $A$ in $U$, i.e., $\operatorname{supp} A=\{u \in U \mid A(u)>0\}$, and we define the cardinality $|A|$ of a fuzzy set $A$ in $U$ as $|A|=\sum_{u \in U} A(u)$. Note also that we reuse the name of a crisp set for its characteristic mapping. In particular, $U$ denotes both the universe and the mapping that associates 1 with each element from the universe, and $\emptyset$ denotes the empty set as well as the $U \rightarrow[0,1]$ mapping given by $\emptyset(u)=0$, for all $u \in U$.

\subsection{Conjunctors, copulas, and t-norms}

A conjunctor is an increasing $[0,1]^{2} \rightarrow[0,1]$ mapping $\mathcal{C}$ that satisfies $\mathcal{C}(0,0)=$ $\mathcal{C}(0,1)=\mathcal{C}(1,0)=0$ and $\mathcal{C}(1,1)=1$, i.e., that coincides with the Boolean conjunction on $\{0,1\}^{2}$. The following potential properties of a conjunctor $\mathcal{C}$ are interesting:

$\mathcal{C}(x, 1)=\mathcal{C}(1, x)=x \quad$ (1 neutral) 
Only the first pages of this paper are presented here because of copyright restrictions. If you would like to obtain the full text, please e-mail to Klaas.Bosteels@UGent.be. 